\title{
ON THE CAUSES, CONSEQUENCES, AND TREATMENT OF INFLAMMATION OF THE VEINS.
}

By HENRY LEE, Esq., F.R.C.S.Fng., Assistant-Surgeon to King's College Hospital, and Surgeon to the Lock Hospital.

(Concluded from $p .503$ of last Number.)

THE term Phlebitis, originally restricted to designate inflammation of one of the larger veins of the body, has gradually been extended so as to include all the consequences which are liable to result from it; and the idea promulgated by $M$. Cruveilhier, that all such secondary affections have their origin in inflammation of the capillary veins of parts at a distance from the original lesion, has been the means of gradually associating the term rather with the grave constitutional affections arising from a poisoned state of the blood, than with any local disease.

In my last paper, I considered some of the effects likely to arise in different capillary systems from the stimulating nature of their contents, and from the mechanical impediment to the circulation produced by the admixture of foreign substances. At present, I shall in like manner review some of the results produced by the presence in the blood of substances which have a tendency to alter its condition, either by producing its coagulation, or by causing its separation into its different parts.

III. Since the time of Ambroise Paré, different surgeons have, with greater or less accuracy, described the abscesses which form in the lungs and liver after injuries to other parts. These abscesses were observed to be peculiar, in the sudden and unexpected manner in which they make their appearance, and also in the circumstance of several of them forming at the same time, leaving the intervening structure of the organ in its natural condition. The want of knowledge of the real way in which they originate, was veiled by ascribing their formation to " sympathetic action", "metastasis", "constitutional irritation", etc , But as such words have been used without any distinct meaning, their place might, perhaps, be advantageously supplied by the term " translation", employed to indicate that the materies morbi is transferred from one part of the body to another, in the manner and under the circumstances which have been already pointed out.

John Hunter was the first, in modern times, to study the actual conditions under which abscesses formed in veins. ${ }^{1}$ His observations proved that the internal coats of veins, like the internal surfaces of serous cavities, might become the seat of inflammation, and that such inflammation might terminate in suppuration. "From attentive examination of abscesses of the lungs, he found many appearances which served to confirm this supposition. He often observed the internal surface of veins leading from such abscesses, not only in an inflamed state, but, in many places, suppurated; and he could even distinctly trace the progresseand termination both of the inflammation and sup. puration."

1 Medical and Philosophical Commentaries by a Society in Edinburgh, vol. iii. 
It is not a little remarkable, that Hunter, having so closely traced the progress of inflammation of the larger veins in the extremities, and also of the smaller veins in the lungs, should have failed to recognise the relation which these so frequently have to each other. Almost all pathologists who have written upon inflammation of the veins, have, in fact, taken for granted, that the injurious consequences produced were necessarily transmitted by continuity of surface. It was therefore natural enough, that the investigation of the effects of inflammation of the veins should terminate with the appearances of irritation of their lining membrane; and as these could rarely be traced in the direction of the circulation so far as the heart, and never beyond it, the consideration of the disease naturally terminated at this point.

In the dissections which Hunter made, he usually found the abscesses which had formed in the course of the veins accurately circumscribed, being separated from the fluid blood by firm coagula. $\mathrm{He}$, however, showed, in a preparation still preserved in the College of Surgeons, that the coagulum might be absent upon one side, and then, he conceived, the purulent secretion might become mixed with the blood. What the circumstances were which determined the formation of coagula in one case, and not in another, Mr. Hunter did not attempt to define; but the following case which he has left on record, leaves no doubt with regard to his having duly appreciated the important office which they perform.

Case xuII. A man was taken into St. George's Hospital with an inflammation of the arm, in consequence of having been bled in the basilic vein. He died suddenly at the expiration of eight days. Upon examination, the vein was found inflamed; the inflammation extended from the puncture which had been made by the lancet as high as the axilla; it went also some way down below the puncture. The orifice where the lancet had been introduced was yet open, and a probe could easily be made to pass through it. The coats of the vein were much thickened, and even the coats of the artery were affected in consequence of its vicinity to the diseased part. About the middle of the arm the vein had suppurated, and was divided by the abscess into two. Each extremity, like the internal surface of the abscess, was irregular and jagged. From the cavity of the abscess there was a free passage into the axillary vein. The cause of the patient's death was attributed to some accidental change in the position of the arm, by which the purulent matter was mixed with the refluent blood, and immediately carried to the heart.

In the dissection of horses which had died after bleeding, Mr. Hunter had traced the inflammation to the heart. To this he attributed the death ; thereby giving origin to the notion, since so generally received, that the fatal symptoms arose from the extension of inflammation in a continuous and uninterrupted manner along the lining membrane of the vessels. In dissenting from this opinion, which has been followed by the very great majority of subsequent writers, it is necessary to remark, that in some of the most rapidly fatal instances, the inflammation has not extended far along the veins; and Mr. Arnott has, by a very careful collection of cases, clearly shown that it by no means generally extends to the heart.

It appears to us a most important fact in connexion with the patho- 
logy of this disease, that wherever a coagulum is found to have occupied a vein for any length of time, there the coats of the vessel will be found thickened; but that, on the contrary, no change will be found to have taken place where the circulation has remained unobstructed.

In examining the veins of those who die of phlebitis, it is not at all uncommon to see such portions of them as have been blocked up with coagula, thickened by adhesive inflammation, while intermediate portions (which have contained no coagula, or where these have been only recently formed) remain in their natural condition. Specimens illustrating this point may be seen among Dr. R. Lee's preparations in the museum of St. George's Hospital; and the evidence which they afford is not the less satisfactory from the dissections having been made to confirm other and different opinions.

It must be evident, in such cases, that it is the stagnation of the vitiated blood in contact with the lining membrane of the vein, which produces the inflammation; and we submit, that it matters not at what intervals such stagnation may take place. The essential characters of the disease are the same, whether the poisoned blood coagulates through the whole of a vein, or in its different parts; whether in the vessel in which the vitiated secretion is first formed, or in some distant system of capillaries. It is, in fact, the poisoned condition of the blood, and not the extension of the inflammation to any particular part, which gives rise to the severe constitutional symptoms. The inflammation which is set up in internal organs, after blecding, thus appears to have the same essential characters as that which affects the vein originally wounded. The disease is merely translated from one part of the venous system to another; and we have thus no difficulty in accounting for the multiform character of the secondary affections, of which, otherwise, it would be difficult to offer any rational explanation.

It is important, in the elucidation of the symptoms of this disease, that we distinguish between the coagula formed in veins by vitiated and by healthy blood. The former will irritate and inflame the coats of the veins; while the latter forms the most efficient barrier against the extension of the inflammation. In examining the veins of those who have died of phlebitis, it will sometimes happen that the lining membrane will appear "blood stained". This may occur either with or without any thickening of the coats of the vessel. It is not difficult to account for this appcarance when a coagulum has remained in contact with the discoloured membrane; but it is occasionally observed where no coagulum is found upon dissection. It is extremely probable, in such cases, that the appearance is still due to a coagulum of blood having for some time occupied the vessel, and having subsequently been removed, either by the natural force of the circulation, or by accidental causes.

The following case from the Hunterian MSS. (Cases and Dissections) may illustrate this point :

Case xuIII. Mr. - received a blow on the right side of the face, which stunned him, but from the immediate effects of which he soon recovered. He was bled; but the opening in the arm opened, and he bled again. An inflammation came on in the arm, which was very severe; and a suppuration at the orifice took place. He was taken with stupor, sleepiness, and sickness at the stomach. It was, 
at the time, doubted whether these symptoms depended upon the blow he had received, or upon the inflammation of the arm. He continued in much the same state until the twelfth day, when he was seized with a most violent shivering fit. He became as cold as possible, subsequently hot, and then covered by a profuse perspiration. He remained all day very low and debilitated, and his arm had less of the fluid red colour than previously. On the fifteenth day he became, in some degree, comatose, lost the power of speech, and died a day or two afterwards.

The preparation of the veins taken from this case, and preserved in the College, shows the cephalic distended through nearly its whole course by a firm clot. This, at the upper part, becomes smaller, and tapers to a narrow flat band, which adheres firmly, by one of its surfaces, to the adjacent wall of the vein. The walls of the vein at this and at the lower part appear healthy; but, in the intermediate portion, they are thick and tough. The median-cephalic and median-basilic veins, and the lower part of the basilic vein, are similarly filled with firm round clots. The upper part of the basilic vein is pervious; but its inner coat is discoloured, being blood-stained. An attentive examination of this preparation will, I belicve, leave little doubt that the discoloration must have been produced by the contact of a coagulum, which, for a certain time, occupied the cavity of the vessel; and it is not improbable that the period of its dissolution, and its removal into the circulation, was marked by the grave accession of constitutional symptoms, which manifested themselves on the twelfth day. The intensity of the local and constitutional symptoms may not unfrequently bear an inverse proportion to each other. In the case just mentioned, the inflammation of the arm became less apparent at the very time that the really serious symptoms first appeared; and, on the other hand, the following case will show that the constitution may remain comparatively unaffected, even in the severest cascs of local phlebitis.

Case xuIv. Thomas Baker, a powerful man, of irregular and intemperate habits, was bled in the left arm, on the 28th of March 1851, having, a few hours previously, been thrown from a horse. He had been stunned at the time of the accident, had broken his collar-bone of the right side, and sustained other injuries about the head and chest. On the 7th of April, the wound made in bleeding, shewed a disposition to suppurate; a pad was, at this time, placed upon the vein, above the wound, and retained in position by a moderately tight bandage. The inflammation about the orifice now subsided, the wound healed, and no further attention was, for the time, paid to it. Ten days after this, the bandage from his arm having been removed, he got upon horseback. As the right arm was still disabled, from the fracture of the collar-bone, he was obliged to use the arm in which he had been bled freely. On returning home, he complained that his ride had "shaken" him.

On the evening of the 19 th, a sharp pricking and smarting sensation was experienced about the upper and inner part of the arm. He felt uncomfortably warm, and, of his own accord, loosened the bandages that were about his shoulders. On the 22 nd, a vivid red patch of inflammation, the size of the palm of the hand, had formed upon the upper and inner part of the arm. This was of a bright red colour, 
becoming white for a moment upon pressure. Its outline was, in soine parts, tolerably well defined; but, in others, it faded gradually into the colour of the surrounding parts. The inflamed part was very hot to the touch, and cxquisitely painful upon pressure. The pulse was quick and full, but not hard; the skin hot, but not dry; the tongue coated with a yellowish brown fur, which was not unusual with him; and the bowels had been freely opened by medicine. The cicatrix of the wound made in bleeding, appeared perfectly well healed, and showed no signs of irritation. A pad was carefully adjusted, so as to make slight pressure over the upper part of the basilic vein; its lower border corresponded with the termination of the redness in the arm. He was ordered bark, with some liquor ammonia acetatis.

During the two following days, the inflammation extended downwards, in the course of the vessels, and indicated some disposition to spread to the back of the arm. He now continued the bark alone. On the 25th, the redness had extended below the elbow; several large vesicles had formed upon the arm, principally in the direction of the basilic vein; the cicatrix at the bend of the elbow discharged some dark coloured matter, -its edges were tumid and everted; the whole of the inflamed parts were exquisitely sensitive; pulse 108, compressible ; he had some comfortable sleep. From this period, the inflammation gradually spread, involving the whole of the arm; the skin lost its bright red character, and became more livid, and abscesses formed slowly in succession, in every part. The inflammation, which spread so extensively in other directions, nerer encroached upon the situation of the pad, which had been placed over the upper part of the basilic vein. At the time when the inflammation was most intense, the patient became delirious for three or four nights; but this probably depended upon his having been denied his usual quantity of stimulus. With this exception, there occurred no symptoms to indicate that any vitiated fluid had entered the circulation. In this case no shivering occurred, either at its commencement, or at any other period; thus affording a strong contrast to the case last mentioned.

The extent to which the blood may coagulate in the living body, in similar cases, is remarkable. When the whole diameter of a vein is obstructed at any one point, a mechanical impediment is necessarily afforded to the circulation in the smaller veins which supply it. The blood, then, is placed under circumstances favourable to its coagulation in them also; and the same disposition is induced in them as in the larger vessels. The obstruction thus produced in veins will account for the tendency evinced, in some cases, for the inflammation to spread in a direction contrary to the natural course of the circulation; whereas, if no such obstruction exists, it will follow the course of the blood. It is evident that, under these circumstances, it may become an important object in the treatment to place a limb under those conditions which may favour the formation of a coagulum in the affected vein. The local inflammation may, by this means, be increased; but the constitutional affection is prevented. However severe the local symptoms may be, if there be fibrine enough in the blood to form a coagulum, and seal the vessel, the patient is comparatively safe. This observation applies with even greater force to cases of open or divided veins; such as present themselves after childbirth, vor. III. 
on the inner surface of the uterus; or, after amputation, on the suppurating surfaces of stumps.

IV. The difference between the effects produced by the introduction of foreign substances into the blood, when they are retained in the first system of capillaries to which they are conveyed, and those which result when they are allowed to circulate with the blood, is strikingly illustrated by the following experiments.

EXPERIMENT XXIX. Fifteen grains of crystallized acetate of lead, dissolved without decomposition in rain water, were injected (a third part at a time) into the crural artery of a large dog. Each injection caused very great pain. The limb, after the operation, became completely paralytic, relaxed, and cold. The pulse and respiration were frequent, and there was a good deal of thirst. In the evening, the limb had swollen, but did not in the least recover its heat, its sensibility, nor its motion. The pain continued unabated. The animal died twelve hours after the experiment.

Post-mortem Appearances. All the organs in the interior of the body were healthy; but the limb was much swollen, emphysematous, and infiltrated with reddish serum. The muscles had become of a pulpy consistence, and of a reddish-black colour. The lighter structures had assumed a violet colour; and the whole limb had the appearance of having been affected with gangrenous inflammation.

EXPERIM ENT XXX. Four grains of tartar emetic, dissolved in an ounce of water, were injected into the crural artery of a large dog. Some pain was apparently experienced during this operation; and the animal subsequently walked a little lame upon the affected leg. From the time of the operation, all food was refused; and violent retching and vomiting came on at the expiration of a quarter of an hour. Much discomfort and restlessness were now apparent. The pulse was small and feverish; but the limb, when handled, in no wise appeared preternaturally sensible (affording in this respect a strong contrast with the limb in the experiment last mentioned). An hour and a quarter after the operation, a kind of diarrhœa came on, accompanied by a repetition of the vomiting. These symptoms recurred occasionally until about three hours after the injection of the fluid, when the animal died.

Post-mortem Appearances. The limb upon which the experiment had been performed presented nothing unusual. The peritoneum was slightly injected, and contained some reddish serum. The stomach also was slightly injected, but only at its pyloric extremity. The mucous membrane of the duodenum throughout was livid, of the colour of the lees of red wine. This appearance was continued into the jejunum; but the other portions of the alimentary canal were apparently healthy. The liver was congested, and mottled upon its convex surface. In some situations its structure was so altered in consistence, as to break down upon very slight pressure. The gallbladder was healthy, and full of bile, which was neither thick nor dark coloured. The lungs were studded here and there with a considerable number of petechial spots, or small black patches.

Direct experiment, in these instances, affords us the same information which we obtain from the more lengthened observation of cases as they occur in practice; viz. that foreign substances, when introduced 
into the blood, in some cases being retained in the part, expend their influence in producing a local, although perhaps a most severe, disease; while in other instances, circulating more freely with the blood, they leave no trace of their entrance into the system, but produce a strictly constitutional affection.

The diseases which we have now to consider, belong principally to the last-mentioned class. It must, however, be remembered, that the two sets of symptoms continually present themselves, in every shade of intensity, in the same case ; and that infected blood, which at one period of the disease goes the round of the circulation, may at another be arrested in some system of capillaries, and give rise to an apparently local disease.

Experinent XXXr. An ounce of fluid, resulting from the fermentation of some leaves of beet-root, in about an equal quantity of water, was injected into the jugular vein of a very little dog. At the expiration of an hour, the animal vomited. This was followed by evacuations of the bowels, at first of moderate consistence, but subsequently liquid, and consisting chiefly of mucus. Symptoms of dysentery now made their appearance. There was pain in the stomach upon pressure, difficulty of breathing, and expression of much uneasiness. Three hours after the injection, there were complete prostration of strength, inability to walk, or even to stand; and fresh evacuations of gelatinous mucus, tinged with blood. These symptoms were followed by renewed vomiting of glairy bilious fluid, and discharge of urine. The fluid evacuations from the bowels were now repeated upwards of five times, and were very fetid, tinged with blood, and black as if mixed with soot. Under the persistence of these symptoms, the strength became greatly impaired. The animal was affected with convulsive twitchings of the muscles, and died eleven hours after the injection.

Post-mortem Appearances. The body was opened while yet warm. The lungs were found somewhat dark and congested, but free from inflammation or petechiæ. The intestinal canal, from the pylorus to the anus, was covered with a muco-sanguineous fluid, resembling the lees of red wine. The mucous membrane of the intestine was, however, everywhere healthy, except in the duodenum, the commencement of the jejunum, and the rectum. In these situations it was of a violet red colour, and bore marks of having been inflamed in longitudinal ridges.

EXPERIMENT XXXIr. Some becf and blood were allowed to decompose in water, at a temperature of about $80 \mathrm{Fahr}$. Five ounces of this fluid, which was quite putrid, were injected in divided quantities into the peritoneal cavity of a small dog. After the experiment, there was prostration of strength, vomiting, discharge of fæces with tenesmus, and increased sensibility of the stomach. At the expiration of an hour, the vomiting and evacuations, consisting of gelatinous mucus, recurred several times. The animal died nine hours after the operation.

Post-mortem Appearances. About a pint and a half of bloody serum was found in the peritoneal cavity. This was without smell, and of the colour of the lees of wine. The peritoneum was inflamed, of a violet red colour, and presented everywhere patches of ecchymosis or congestion. The inflammation appeared to have been very great, and was accompanied by the formation of dark patches along the course of 
the vessels contained in the mesentery. The discolorations were also found at the attached margin of the intestine. The whole of the mucous membrane of the intestinal canal, from the stomach to the anus, was highly inflamed, and of a deep red, violet, or black colour. The folds of mucous membrane which projected into the cavity of the intestine were most discoloured; and in the stomach, these were the only parts inflamed. A glutinous but not bloody mucus was contained in the intestines. The muscular layer appeared in no way to have participated in the affection. The bladder was empty and contracted; it was inflamed on its peritoneal surface, but of its natural appearance within. The left pleura contained some bloody serum. The spleen and the lungs were studded with patches of congestion.

Experiment xxxiII. The last experiment was repeated on a large dog. Two ounces and a half of fluid, obtained from putrid beef, not so thick or fetid as in the preceding case, were thrown into the peritoneal cavity. Very great pain was immediately produced. This was followed by prostration of strength, aversion to food, fever without vomiting, alvine evacuations, and death at the end of ten hours.

Post-mortem Appearances. About two pints of bloody serum were found in the peritoneal cavity. The peritoneum itself was excessively inflamed, of a violet red colour, studded with black patches, and presenting here and there inflammatory adhesions and exudations. The bladder was of a deep red colour externally, but quite of its natural appearance within. The intestines were inflamed on their peritoneal surface, but still more so internally. The mucous membrane, from the pylorus to the anus, was thickened, of the colour of the lees of wine, and coated with mucus. Even those portions of the intestines which (in consequence of having been protected from the contact of the morbid fluid) were not inflamed on their serous surface, were nevertheless affected internally.

The general result of the presence of putrid fluids in the system, whether introduced directly into the circulation, or by absorption from serous surfaces, is, as evinced in the foregoing cases, to produce a remarkable affection, peculiarly characterised by congestion of the mucous membrane of the intestines. The evacuations that accompany this condition, are evidently an effort of nature to relieve the system from the vitiated fluids which have entered the circulation; and it is not a little remarkable, that the mucous membrane of the intestincs should in these cases be exclusively affected. The appearances produced may be distinguished from the results of inflammation, in that no thickening or shrinking of the tissues is produced; but they are swollen, congested, and blood-stained, either in petechial spots, in larger patches, or over a continuous surface. The discharge from the intestines, in such cases, consists chiefly of mucus; but this may sometimes be accompanied by a kind of passive hæmorrhage, and occasionally the secretion may assume a puriform character, without any abrasion of the mucous lining of the canal.

We can readily understand, under these circumstances, that the appearances and symptoms which, a few years ago, were so often observed and described as forming a separate disease, under the name of gastro-enteritis, may frequently have been only the secondary results produced by an unhealthy condition of the blood. 
When the actions produced by the introduction of putrid fluids into the body are fully developed, all the indications of putrefaction may take place even in the living body. Not only the fluids, but the socalled solids, pass rapidly into decomposition; and the former would appear, in addition to being extremely liable to decompose themselves, to have the property, under certain circumstances, of infecting other parts, and even other healthy bodies. Thus, we read, that during the plague of Marseilles, the bile taken from those who had died of the disease, uniformly produced death when injected into the veins of dogs.

After a putrid element has been introduced into the system, the blood is evidently altered in composition. It becomes black, viscid, and, in a great measure, deprived of its fibrine. After death, it is found fluid, of a very dark colour, and not unfrequently mixed with gas.

It might be, and, perhaps, generally is supposed, that putrid fluids mixed with the blood have the power of preventing its coagulation. The experiments which I have performed on this subject have, however, led me to an opposite conclusion. I have found that the addition of putrid fluids to healthy blood recently drawn promotes its coagulation. It is nevertheless true, that whenever there is manifested a tendency in the body to decomposition, the blood will be found to have lost its power of coagulation; but this effect arises from the continued eirculation of vitiated fluid with the blood, and is not the immediate result of their admixture. It is probable, in this as in the former classes of cases, that the foreign substance gradually abstracts the fibrine of the blood, giving rise to small petechial spots, or small patches of congestion in different parts; and whatever may be the coagulating power of the blood when the putrid element is first introduced, it rapidly becomes impaired, or altogether lost.

Of all the substances with which the experiments have been tried, none possesses the power of coagulating blood so rapidly and so completely as pus. To this fact we attribute the formation of the larger patches of congestion found in internal organs when pus has accidentally entered the circulation; and with the more feeble influence of putrid fluids, or of mercury, we naturally associate the formation of the smaller and more circumscribed congestions, where these have given origin to the disease.

Two principal indications arise from the physiological analysis of these affections. First, in phlebitis, strictly so called, there may be, and generally is, a twofold disease, namely, a local inflammation, and a constitutional infection. These, although they occur together, give rise to very different symptoms, and may require very different plans of treatment. It may even be strictly in accordance with the soundest principles of pathology, to use remedies to subdue the violence of the local inflammation, at the same time that tonics are administered to support the general strength of the system. Secondly, we learn, by direct experiment, that where a putrid element is introduced into the circulation, the mucous membrane of the intestines and the liver are the principal channels by means of which the elimination of the morbid matter is attempted. The functions of the intestines would appear to fit them in a peculiar manner for this office; and it is probably some power connected with their natural action which determines so large a 
flow of vitiated blood to their mucous lining. The congestions which arise in other parts, in consequence of a direct infection of the blood, may terminate in the effusion of serum, lymph, pus, or blood, either separately or mixed together in various proportions. In some rare instances, the secondary inflammation may terminate in resolution; of this the following case affords an instructive example.

CASE XIV. ${ }^{1}$ G. B., æt. 31, was admitted into St. George's Hospital for an aneurism in the left groin, for which the external iliac artery was tied. On the evening after the operation, the whole abdomen became painful and tender; the pulse was frequent, the skin hot, and the tongue dry and brown. The patient was bled, and on the following day the bleeding was repeated. The adhesions of the edges of the wound were destroyed, and gave exit to some sanious matter contained within. On the third day, none of the local symptoms were relieved, and the constitutional symptoms were much aggravated. The right shoulder now became affected with intense pain, accompanied by swelling and tenderness. Half a pint of wine was prescribed to be taken daily, with such nourishment as the stomach could receive. The pain in the shoulder, with some degree of swelling, continued for some time; but on the quantity of the stimulus being farther increased, this, as well as the other symptoms, gradually subsided.

This case is interesting, not only as showing that a secondary inflammation may terminate in resolution, but also as indicating the kind of constitution in which such secondary affections are likely to occur. Three months after the patient was discharged from the hospital as cured, the pulsation returned in the tumour, which again enlarged to a very considerable size. It is therefore evident that the coagulating power of his blood had from some cause been greatly impaired : for had this not been the case, the coagula which had been allowed such ample time to form, would have permanently closed the dilated vessel.

13, Dover Street, Piocadilly, June 1851.

\section{HYSTERICAL AFFECTIONS OF THE HIP JOINT.}

By WILLIAM COULSON, Esq., F.R.S.C.Eng., Surgeon to St. Mary's Hospital ; and to the Magdulen Hospital, etc.

I was consulted in the case of a young lady, nineteen years of age, pale and delicate looking, of highly nervous and excitable temperament, on account of an affection of the right hip joint, for which she had been under surgical treatment, and for a considerable time confined to the recumbent position. The frequent application of leeches, and the employment of counter-irritation in every possible form, had proved without avail ; she lay helpless and bed-ridden, complaining of severe pain extending over the whole lower extremity, and impressed with a firm conviction that she would remain a cripple for life. She shrunk from the slightest attempt to examine the limb, declaring that the pain she experienced was insupportable. My attention, however, was

1 Related by Sir Benjamin C. Brodie in the Medical Gazette. 